www.jmscr.igmpublication.org

Index Copernicus Value: 79.54

ISSN (e)-2347-176x ISSN (p) 2455-0450

crossref DOI: https://dx.doi.org/10.18535/jmscr/v7i4.115

\title{
A Case of Recurrent Ameloblastoma with histopathological diversity in the infratemporal fossa region
}

\author{
Authors \\ Narendra Babu BK*, Sudip Indu, Sudharshan Bhat, Aditi Rawat \\ Indian Army Dental Corps \\ *Corresponding Author \\ Narendra Babu BK \\ Email: babuosmf75@gmail.com
}

\begin{abstract}
A 52yrs/f was referred to our centre, with complaints of a progressively enlarging nontender swelling in the left side upper jaw, past six months. Medical history revealed history of three surgeries, the last surgery performed was hemimandibulectomy left side in 1999. Intraoral examination revealed solitary oval shaped swelling measuring approx $2 \times 1.5 \mathrm{cms}$ near the maxillary tuberosity region. The swelling was firm and non tender on palpation. The non contrast computed tomography (NCCT) revealed a multilocular radiolucent lesion extending in to the infratemporal fossa. Incisional biopsy examination was suggestive of acanthomatous and granular variety of Ameloblastoma. Modified Alkayat Bramley approach was used and an access osteotomy of zygomatic arch was done, the mass was removed in total under general anaesthesia. Post operative recovery was uneventful with a satisfactory functional and aesthetic outcome.

Keywords: Ameloblastoma, Acanthomatous, Follicular, Granular, Maxillary tuberosity, Recurrence, Infra temporal fossa, Access Osteotomy, Zygomatic arch.
\end{abstract}

\section{Introduction}

Malassez in 1885 coined the word adamantinoma to signify the odontogenic tumor which had been recognized by Cusack in 1827 and described later by Falksson ${ }^{[1,2,3]}$. Later Ivey and Churchill in 1930 introduced the term ameloblastoma ${ }^{[4]}$. The literature describes ameloblastoma as benign neoplasm which is invasive in nature, consisting of proliferating odontogenic epithelium supported by fibrous stroma. Hinds et al. ${ }^{[5]}$ and Gorlin et a1. ${ }^{[6]}$ collected specimens and studied them suggesting the probable orgin of cells forming these lesions are epithelial lining of an odontogenic cyst, dental lamina or enamel organ, stratified squamous epithelium of the oral cavity, displaced dental epithelial remnants tissues without bone involvement. Smith ${ }^{[7]}$ described that the tumor can arise from basal cells of the oral epithelium or from cells that have undergone differentiation to imitate as ameloblasts. Ameloblastoma can represent in an array of clinical, radiological and histological forms, its aggressive nature with its ability to cause widespread destruction of jaws with penetration into the surrounding soft tissues makes the management challenging. Even though they are rare and comprise 10 percentages of odontogenic tumors and cysts. The lesion has always been a 
topic of extensive debate in terms of their varied histopathological diversity and local recurrence following surgical treatment. The literature cites its rare presentation in infratemporal fossa, orbit, and anterior skull base ${ }^{[8,9,10]}$. The purpose of this case report is to highlight the inadequacy in treatment resulting in its loco regional recurrence.

\section{Case Report}

A 52yrs/f was referred to our centre in 2018, with complaints of a progressively enlarging nontender swelling in the left side upper jaw, past six months. Medical history revealed history of three surgeries; the last surgery consisted of hemimandibulectomy lt side in 1999 with a different diagnosis of same lesion, calcifying epithelial odontogenic tumour and odontogenic keratocyst.

On clinical examination, there was a obvious facial asymmetry, a diffuse ill defined swelling on the left side of face $2 \mathrm{~cm}$ anterior to the tragus, over lying skin was normal, nontender on palpation, firm in consistency, restricted mandibular movements, deviation of the lower jaw towards the left on opening, interincisal opening $35 \mathrm{~mm}$. Intraoral examination revealed solitary oval shaped swelling measuring approx 2 x $1.5 \mathrm{cms}$ near the maxillary tuberosity region (Lt) obliterating the maxillary buccal vestibule $(\mathrm{Lt})$. The swelling was firm and non tender on palpation. Overlying mucosa was blanched Fig.no.1.

On radiological examination, computed tomography axial section Fig.no.2 revealed a well-defined multilocular radiolucency in the infratemporal region medial to the left zygomatic arch, approx $3 \mathrm{~cm} \times 2 \mathrm{~cm}$. At the centre of the lesion a well defined linear radioapacity was seen Fig.no 3, suggestive of coronoid process left back in the past surgery.

Incisional biopsy was reported as solid multicystic ameloblastoma with acanthomatous and granular cell changes.

On clinical, radiological and histopathological examination, it was confirmed as a case of recurrent ameloblastoma of mandible. The case was worked up for excision under general anaesthesia. As the nidus of the lesion was probably the coronoid process, the surgery was focussed towards complete removal of mass along with the coronoid. After meticulous preoperative work-up, patient underwent complete excision of the mass in the infratemporal fossa, To facilitate this an access osteotomy of zygomatic arch, was carried out Fig.no.3, a collective modified Alkyat Bramley and vestibular incision was used. The specimen Fig.no.4 was submitted for histopathological examination and was confirmed as solid multicystic ameloblastoma with predominantly follicular pattern along with acanthomatous and granular Cell Changes. Postoperative recovery was uneventful. The patient is under follow up and has not exhibited recurrence.

\section{Histological Findings}

The histopathology of the section was consistent with the incisional biopsy report of ameloblastoma. Microscopy revealed islands of ameloblastic follicles with central stellate reticulum like cells and peripheral ameloblast like cells. The nuclei of pre ameloblasts were polarized away from the basement membrane. Few of the follicles exhibited squamous metaplasia and granular cell changes. The stroma was mature collagenized in nature. The final diagnosis was rendered as ameloblastoma with predominantly follicular pattern along with acanthomatous and granular cell changes Fig. no.5.

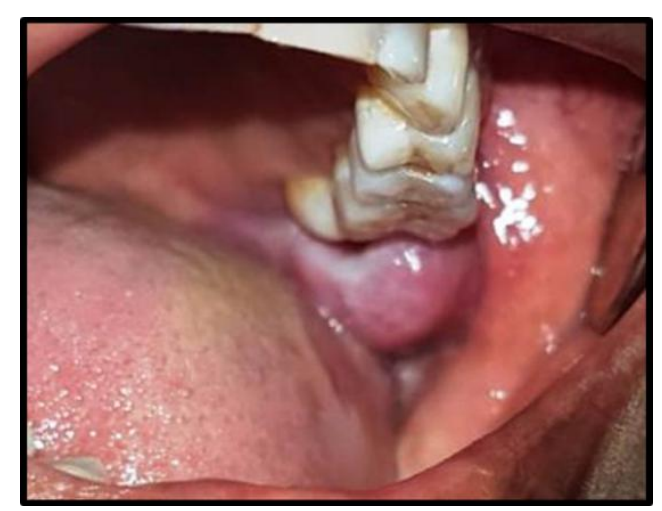

Figure.no.1 


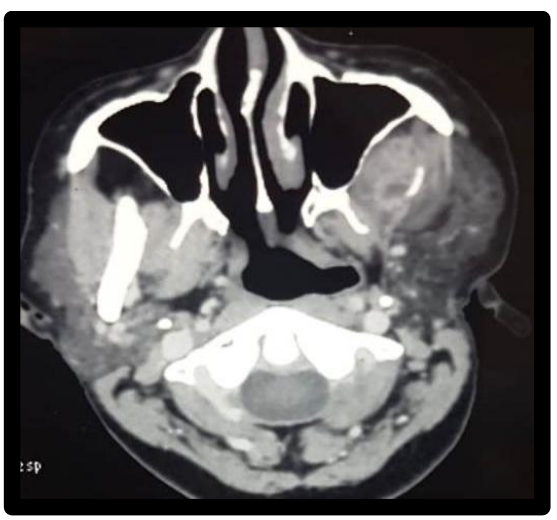

Figure.no.2

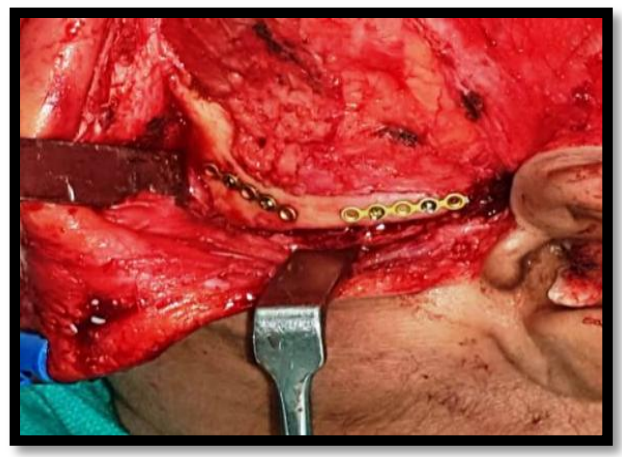

Figure.no.3

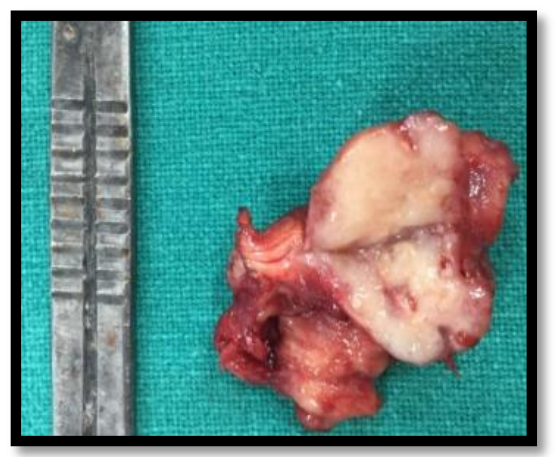

Figure.no.4

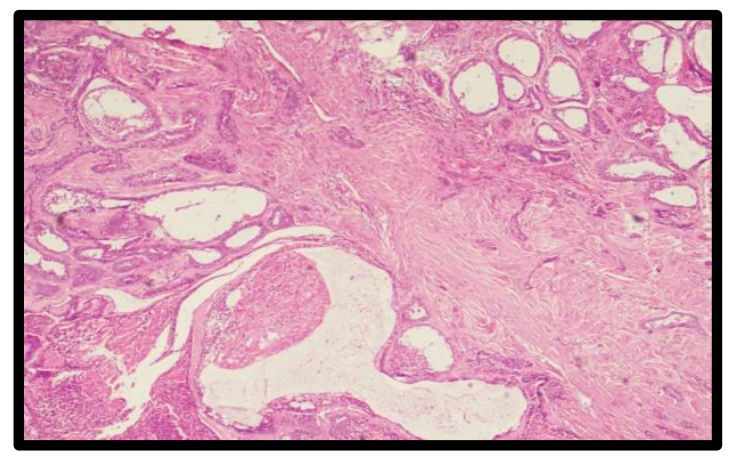

Figure.no.5

\section{Discussion}

The WHO 1992, describes, ameloblastoma as a benign but locally invasive polymorphic neoplasm consisting of proliferating odontogenic epithelium, which usually has a follicular or plexiform pattern, lying in a fibrous stroma. The tumour represents approx $1 \%$ of all mandibular and maxillary tumours and cysts and approximately $10 \%$ of all odontogenic mandibular tumours, Philipsen and Reichart, 2002 ${ }^{(11)}$. The literature reveals the age of occurrence being the 3 rd and 5th decade. The mandible typically is affected five times more frequently than the maxilla. (Robinson and Martinez, 1977; Eversole et al., 1984). The tumor is commonly asymptomatic, presenting as a slow growing mass capable of invading loco regional tissues with a high tendency to recur, recurrence rate being 5 to $75 \%$ Olaitan et al ${ }^{(12)}, 1993$; Reichart et al ${ }^{(13)}, 1995$. Recurrence is dependent on the method of treatment, the type and the size of the ameloblastoma, and its location. Solid types are said to have a higher risk of recurrence due to their micro-extensions into the surrounding bone (Collins and Harrison, 1993), which holds true for multicystic ameloblastomas, typically multilocular in radiographic appearance, macroscopically cystic nature make it a distinguishable entity. The recurrence pattern, clinical, and radiological presentation in our case was in consensus with the existing literature.

Multicystic ameloblastoma usually appears very similar to a non-neoplastic odontogenic cyst and is frequently clinically misdiagnosed as dentigerous cyst and odontogenic keratocyst, hence histological confirmation is mandatory. This was true in our case; the case was diagnosed as calcifying epithelial odontogenic tumour and enucleation was done. There was recurrence again and diagnosed as odontogenic keratocyst and an hemimandibulectomy was done, third time when it recurred it was seen arising from coronoid process, suggesting that the recurrence was probably due to the pathological fracture of coronoid failure to remove coronoid in previous surgery, resulting in displacement to infratemporal fossa.

Robinson and Martinez (1977), Philipsen HP, Reichart PA differentiated the unicystic from the 
multicystic or solid ameloblastomas, this histomorphological differentiation was added to the existent histological classification Ackermann et al. (plexiform, follicular, desmoplastic, basal, granular.) A range of these histologic subtypes of solid and multicystic ameloblastomas has been cited in the literature, perplexing the surgeon. The variations are basically of academic interest, allowing the pathologist an approval of this histologic diversity. Biologic pattern however is not affected by these variations. In general, these tumors are unencapsulated, infiltrating neoplasms, occasionally there may be areas that show well demarcation. The most frequent pattern is follicular and the second being plexiform. It has been proven that the cellular types are more aggressive than the less cellular (Vickers and Gorlin, 1970). This was in consensus with our case presentation, which reoccurred after 09yrs with predominantly follicular pattern along with acanthomatous and granular Cell Changes.

The multicystic ameloblastoma deserves special consideration on the basis of its clinical, radio imaging, histopathology, and its response to treatment. This variant of ameloblastoma is reported to have shown less aggressive behavior than the other variants ameloblastoma. Various treatment modalities for multicystic cystic ameloblastoma have been used ranging from segmental or marginal resection. However, conservative treatments such as enucleation and curettage have also been tried without any sucess. Marsupialization has been tried to reduce the size of the lesion, followed by second stage surgery by few surgeons. The recurrence rate after treatment of a multicystic ameloblastoma ranges from 10 to $25 \%$. There is no adequate evidence to prove which treatment modality is the most effective. Nevertheless most appropriate treatment modality for ameloblastoma is resection with safe margins. Segmental resection produces good results, especially when carried out as a primary treatment. Shatkin and Hoffmeister showed that continued under-treatment of ameloblastomas can lead to unresectable recurrences. A radical approach is necessary to prevent its recurrence.

\section{Conclusion}

Hence the conclusion can be derived that it is disastrous to leave behind any affected part of tissue which may lead to loco regional recurrence in an anatomical inaccessible areas. Thus requiring access osteotomy for their surgical management and thereby increasing surgical morbidity of the procedure. We unambiguously append, ameloblastoma should be appropriately diagnosed histologically and managed aggressively in the first instance to prevent unresectable recurrences.

\section{Reference}

1. Cusack, J. W.: Report of the amputations of portions of the lower jaw. Dublin Hosp. Rec. 4: 1- 38, 1827.

2. Falksson, R.: Zur Kenntnis der Kieferzysten. Virchows Arch. [Pathol. Anat.] 76:504-510, 1879.

3. Malassez, L.: Sur le role des debris epitheliaux paradentaires. Arch. Physiol. Norm. Pathol. 5:309-340; 6:379-449, 1885.

4. Ivey, R. H., and Churchill, H. R.: The need of a standardized surgical and pathological classification of tumors and anomalies of dental origin. Am. Aaoc. Dent. Sch. Trans. 7:240-245, 1930.

5. Gorlin, R. J., Chaudhry, A. P., and Pindborg, J.: Odontogenic tumors. Cancer 14:73-101, 1961.

6. Hinds, E. C., Pleasants, J. E., and Snyder, P. L.: Management of ameloblastoma. Oral Surg. 7: 1169-1 177, 1954

7. Smith, J. F.: The controversial ameloblastoma. Oral Surg. 26:45-75, 1968.

8. Auluck A, Shetty S, Desai R, Mupparapu M. Recurrent ameloblastoma of the infratemporal fossa: Diagnostic 
implications and a review of the literature.

Dentomaxillofac Radiol. 2007;36:416-9.

9. To EW, Tsang WM, Pang PC. Recurrent ameloblastoma presenting in the temporal fossa. Am J Otolaryngol. 2002;23:105

10. Chen WL, Li JS, Yang ZH, Wang JG, Zhang B. Recurrent ameloblastoma of the anterior skull base: Three cases treated by radical resections. J Craniomaxillofac Surg. 2006;34:412-4.

11. Philipsen HP, Reichart PA: Revision of the 1992-edition of the WHO histological typing of odontogenetic tumors. A suggestion. J Oral Pathol Med 31(5): 253258, 2002.

12. Olaitan AA, Adeola DS, Adekeye EO: Ameloblastoma: clinical features and management of 315 cases from Kaduna, Nigeria. J Craniomaxillofac Surg 21: 351355, 1993.

13. Reichart PA, Philipsen HP, Sonner S: Ameloblastoma: a biological profile of 3677 cases. Eur J Cancer B Oral Oncol 31B(2): 86-99, 1995.

14. Leider AS, Eversole LR, Barkin ME. Cystic ameloblastoma. A clinicopathologic analysis. Oral Surg Oral Med Oral Pathol 1985: 60: 624-630. 